\title{
Local Fractional Derivatives of Differentiable Functions are Integer-order Derivatives or Zero
}

\author{
Vasily E. Tarasov 1
}

Published online: 18 April 2015

(C) Springer India Pvt. Ltd. 2015

\begin{abstract}
In this paper we prove that local fractional derivatives of differentiable functions are integer-order derivative or zero operator. We demonstrate that the local fractional derivatives are limits of the left-sided Caputo fractional derivatives. The Caputo derivative of fractional order $\alpha$ of function $f(x)$ is defined as a fractional integration of order $n-\alpha$ of the derivative $f^{(n)}(x)$ of integer order $n$. The requirement of the existence of integer-order derivatives allows us to conclude that the local fractional derivative cannot be considered as the best method to describe nowhere differentiable functions and fractal objects. We also prove that unviolated Leibniz rule cannot hold for derivatives of orders $\alpha \neq 1$.
\end{abstract}

Keywords Fractional derivative $\cdot$ Leibniz rule $\cdot$ Local fractional derivative

Mathematics Subject Classification 26A33 Fractional derivatives and integrals

\section{Introduction}

Fractional calculus as a theory of derivatives and integrals of non-integer orders [1-3] has a long history [4-6]. As a mathematical tool it has a lot of applications in physics and mechanics to to describe media and systems with nonlocal and hereditary properties of power-law type. During more than three hundred years of history of fractional calculus it has been proposed different definitions of fractional derivatives by Riemann, Liouville, Riesz, Grünwald, Letnikov, Marchaud, Caputo [1,2]. Fractional-order derivatives can be characterized by a set of interesting unusual properties such as a violation of the usual Leibniz rule, a deformations of the usual chain rule, a violation of the semi-group property. These unusual properties can be used to describe nontrivial and unusual properties of complex

Vasily E. Tarasov

tarasov@theory.sinp.msu.ru

1 Skobeltsyn Institute of Nuclear Physics, Lomonosov Moscow State University,

Moscow 119991, Russia 
systems and media. Therefore these properties are valuable and important for applications in physics and mechanics (see [9-11] and references therein).

Recently, so-called local fractional derivative has been proposed in [12-15] by a motivation of describing the properties of fractal objects and processes. The definition of the local fractional derivative is obtained from the Riemann-Liouville fractional derivative by introducing two changes such as a subtraction of finite number of terms of the Taylor series and the taking the limit. The motivation of this definition is a studying fractional differentiability properties of nowhere differentiable functions and fractal objects.

In this paper, we demonstrate that the definition of local fractional derivative can be presented as a limit of the Caputo fractional derivatives. We note that the Caputo fractional derivative of order $\alpha(n-1<\alpha \leq n)$ of a function is defined as an action of derivative of integer order $n$ and then the integration of non-integer order. Therefore the function should be differentiable $n$ times. In this paper we also prove that local fractional derivatives of differentiable functions are integer-order derivative or zero operator. We argued that unviolated Leibniz rule cannot hold for local fractional derivatives of orders $\alpha \neq 1$.

\section{Local Fractional Derivative via Caputo Fractional Derivative}

The local fractional derivative $\mathcal{D}^{\alpha}$ of order $\alpha$ of a function $f(x)$ at the point $x=a$ is usually defined (see Eq. 2.6 of [13]) by the equation

$$
\left(\mathcal{D}^{\alpha} f\right)(a):=\lim _{x \rightarrow a}{ }_{a}^{R L} \mathcal{D}_{x}^{\alpha}\left(f(x)-\sum_{k=0}^{n-1} \frac{f^{(k)}(a)}{k !}(x-a)^{k}\right), \quad(n-1<\alpha \leq n),
$$

where ${ }_{a}^{R L} \mathcal{D}_{x}^{\alpha}$ is the left-sided Riemann-Liouville fractional derivative of order $\alpha$ (see Eqs. (2.1.15) and (2.1.1) of [2]) that is defined by

$$
{ }_{a}^{R L} \mathcal{D}_{x}^{\alpha} f(x):=\frac{1}{\Gamma(n-\alpha)} \frac{d^{n}}{d x^{n}} \int_{a}^{x} \frac{f(z) d z}{(x-z)^{\alpha}} . \quad(n-1<\alpha \leq n) .
$$

The Riemann-Liouville fractional derivative ${ }_{a}^{R L} \mathcal{D}_{x}^{\alpha}$ is nonlocal operator. For $0<\alpha \leq 1$, Eqs. (1) and (2) have the forms

$$
\begin{aligned}
\left(\mathcal{D}^{\alpha} f\right)(a) & :=\lim _{x \rightarrow a}{ }_{a}^{R L} \mathcal{D}_{x}^{\alpha}(f(x)-f(a)), \quad(0<\alpha \leq 1), \\
{ }_{a}^{R L} \mathcal{D}_{x}^{\alpha} f(x) & :=\frac{1}{\Gamma(1-\alpha)} \frac{d}{d x} \int_{a}^{x} \frac{f(z) d z}{(x-z)^{\alpha}}, \quad(0<\alpha \leq 1) .
\end{aligned}
$$

In the fractional calculus is well-known the Caputo fractional derivatives [2,3] suggested in [16-19]. The left-sided Caputo fractional derivative of order $\alpha$ can be defined via the above Riemann-Liouville fractional derivative (see Eq. (2.4.1) of [2]) by

$$
\left({ }_{a}^{C} \mathcal{D}_{x}^{\alpha} f\right)(x):={ }_{a}^{R L} \mathcal{D}_{x}^{\alpha}\left(f(x)-\sum_{k=0}^{n-1} \frac{f^{(k)}(a)}{k !}(x-a)^{k}\right), \quad(n-1<\alpha \leq n) .
$$

As a result, the local fractional derivative (1) of order $\alpha$ at the point $x=a$ can be defined via the above Caputo fractional derivative by the equation

$$
\left(\mathcal{D}^{\alpha} f\right)(a):=\lim _{x \rightarrow a}\left({ }_{a}^{C} \mathcal{D}_{x}^{\alpha} f\right)(x), \quad(n-1<\alpha \leq n) .
$$


As a result, we can see that local fractional derivative is a limit of the Caputo fractional derivative.

Proposition The local fractional derivative (1) of order $\alpha$, where $n-1<\alpha \leq n$ and $n \in \mathbb{N}$, at the point $x=x_{0}$ can be represented by the equation

$$
\left(\mathcal{D}^{\alpha} f\right)(a):=\frac{1}{\Gamma(n-\alpha)} \lim _{x \rightarrow a} \int_{a}^{x} \frac{d z}{(x-z)^{\alpha}} \frac{d^{n} f(z)}{d z^{n}}, \quad(n-1<\alpha \leq n) .
$$

Proof This statement is direct corollary of Theorem 2.1 of [2] (see Eq. (2.4.15) of [2]), where the left-sided Caputo fractional derivative is represented in the form

$$
{ }_{a}^{C} \mathcal{D}_{x}^{\alpha} f(x):=\frac{1}{\Gamma(n-\alpha)} \int_{a}^{x} \frac{d z}{(x-z)^{\alpha}} \frac{d^{n} f(z)}{d z^{n}}, \quad(n-1<\alpha \leq n) .
$$

The Caputo fractional derivatives ${ }_{a}^{C} D_{x}^{\alpha}$ can be defined for functions belonging to the space $A C^{n}[a, b]$ of absolutely continuous functions [2]. If $f(x) \in A C^{n}[a, b]$, then the Caputo fractional derivatives exist almost everywhere on $[a, b]$.

It can be directly derived that the left-sided Caputo fractional derivative of the power function $(x-a)^{\beta}$ is

$$
{ }_{a}^{C} \mathcal{D}_{x}^{\alpha}(x-a)^{\beta}=\frac{\Gamma(\beta+1)}{\Gamma(\alpha+\beta+1)}(x-a)^{\beta-\alpha},
$$

where $\beta>-1$ and $\alpha>0$. In particular, if $\beta=0$ and $\alpha>0$, then the Caputo fractional derivative of a constant $C$ is equal to zero:

$$
{ }_{a}^{C} \mathcal{D}_{x}^{\alpha} C=0 .
$$

For $k=0,1,2, \ldots, n-1$, we have

$$
{ }_{a}^{C} \mathcal{D}_{x}^{\alpha}(x-a)^{k}=0 .
$$

It is important to emphasize that the Caputo fractional derivative is represented by Eq. (8) as a sequence of two operations, namely, first taking the ordinary derivative of integer order $n$ and then the integration of fractional order $n-\alpha$, (see Eq. 2.4.17 of [2]):

$$
\left({ }_{a}^{C} \mathcal{D}_{x}^{\alpha} f\right)(x)={ }_{a}^{R L} \mathcal{I}_{x}^{n-\alpha} f^{(n)}(x) .
$$

The Riemann-Liouville fractional derivative is represented by the inverse sequence of the same operations.

\section{Local Fractional Derivative is Derivative of Integer-order}

Let us prove the following statement for the local fractional derivatives of $n$-differentiable functions.

Proposition Local fractional derivative of order $\alpha$, where $n-1<\alpha \leq n, n \in \mathbb{N}$, on a set of $n$-differentiable functions is a derivative of order $n \in \mathbb{N}$ or zero operator. 
Proof If $f(x)$ is a $n$-differentiable function in a neighborhood $U\left(x_{0}\right)$ of $x_{0}$ (i.e., $f(x)$ has a derivative of order $n \in \mathbb{N}$ in a neighborhood of $x_{0}$ ), then the function can be represented by the Taylor series

$$
f(x)=\sum_{k=0}^{n} \frac{f^{(k)}\left(x_{0}\right)}{k !}\left(x-x_{0}\right)^{k}+o\left(\left(x-x_{0}\right)^{n}\right) .
$$

If $f(x)$ is a differentiable function (i.e., $\mathrm{n}=1$ ), then

$$
f(x)=f\left(x_{0}\right)+f^{(1)}\left(x-x_{0}\right)+o\left(\left(x-x_{0}\right)\right) .
$$

For $n$-differentiable functions, we can rewrite the local fractional derivative of order $n-1<$ $\alpha \leq n$ in the form

$$
\begin{aligned}
\left(\mathcal{D}^{\alpha} f\right)\left(x_{0}\right) & =\lim _{x \rightarrow x_{0}}{ }_{x_{0}}^{R L} \mathcal{D}_{x}^{\alpha}\left(f(x)-\sum_{k=0}^{n-1} \frac{f^{(k)}\left(x_{0}\right)}{k !}\left(x-x_{0}\right)^{k}\right), \\
& =\lim _{x \rightarrow x_{0}} x_{0}^{R L} \mathcal{D}_{x}^{\alpha}\left(\frac{f^{(n)}\left(x_{0}\right)}{n !}\left(x-x_{0}\right)^{n}+o\left(\left(x-x_{0}\right)^{n}\right)\right), \\
& =\frac{f^{(n)}\left(x_{0}\right)}{n !} \lim _{x \rightarrow x_{0}}{ }_{x_{0}}^{R L} \mathcal{D}_{x}^{\alpha}\left(\left(x-x_{0}\right)^{n}+o\left(\left(x-x_{0}\right)^{n}\right)\right) .
\end{aligned}
$$

Using $\Gamma(n+1)=n$ ! and the equation (see Property 2.1 of [2])

$$
{ }_{x_{0}}^{R L} \mathcal{D}_{x}^{\alpha}\left(x-x_{0}\right)^{\beta}=\frac{\Gamma(\beta+1)}{\Gamma(\beta+1-\alpha)}\left(x-x_{0}\right)^{\beta-\alpha}, \quad(\beta>-1),
$$

we obtain

$$
\begin{aligned}
\left(\mathcal{D}^{\alpha} f\right)\left(x_{0}\right) & =\frac{f^{(n)}\left(x_{0}\right)}{n !} \lim _{x \rightarrow x_{0}} \frac{\Gamma(n+1)}{\Gamma(n+1-\alpha)}\left(\left(x-x_{0}\right)^{n-\alpha}+o\left(\left(x-x_{0}\right)^{n-\alpha}\right)\right), \\
& =\frac{f^{(n)}\left(x_{0}\right)}{\Gamma(n+1-\alpha)} \lim _{x \rightarrow x_{0}}\left(\left(x-x_{0}\right)^{n-\alpha}+o\left(\left(x-x_{0}\right)^{n-\alpha}\right)\right) .
\end{aligned}
$$

As a result, we get

$$
\left(\mathcal{D}^{\alpha} f\right)\left(x_{0}\right)=\left\{\begin{array}{c}
0 \quad n-1<\alpha<n, \\
f^{(n)}\left(x_{0}\right) \quad \alpha=n,
\end{array}\right.
$$

where $n-1<\alpha \leq n$ and $n \in \mathbb{N}$, and we use $\Gamma(n+1-n)=\Gamma(1)=1$.

The proved statement means that the local fractional derivatives cannot be considered as fractional derivatives on sets of finite (or infinite) numbers of times differentiable functions.

As a corollary of the proved statment we can say that generalizations of the Leibniz rule for local fractional derivatives $\mathcal{D}^{\alpha}$ of order $\alpha>0$ for $n$-differentiable functions $f(x)$ and $g(x)$ should give the relation

$$
\mathcal{D}^{\alpha}(f(x) g(x))=\sum_{k=0}^{n} \frac{\Gamma(\alpha+1)}{\Gamma(\alpha-k+1) \Gamma(k+1)}\left(\mathcal{D}^{\alpha-k} f(x)\right)\left(\mathcal{D}^{k} g(x)\right), \quad(\alpha=n \in \mathbb{N}),
$$

for integer orders $\alpha=n \in \mathbb{N}$ or it should give the equality $0=0$ for noninteger orders $\alpha$, $n-1<\alpha \leq n$. 
For $0<\alpha \leq 1$, Eq. (14) has the form

$$
\left(\mathcal{D}^{\alpha} f\right)\left(x_{0}\right)=\left\{\begin{array}{cc}
0 & 0<\alpha<1 \\
f^{\prime}\left(x_{0}\right) & \alpha=1
\end{array}\right.
$$

Therefore we get the statement that has been proved in [7] as Theorem "No violation of the Leibniz rule. No fractional derivative": If the unviolated Leibniz rule

$$
\mathcal{D}^{\alpha}(f(x) g(x))=\left(\mathcal{D}^{\alpha} f(x)\right) g(x)+f(x)\left(\mathcal{D}^{\alpha} g(x)\right),
$$

holds for the linear operator $\mathcal{D}^{\alpha}$ and the $n$-differentiable functions $f(x)$ and $g(x)$, then this operator is the derivative of integer (first) order, that can be represented as $\mathcal{D}^{\alpha}=a(x) D^{1}$, where $a(x)$ are functions on $\mathbb{R}$.

In paper [7] it was proved that violation of relation (17) is a characteristic property of all types of fractional-order derivatives with $\alpha>0$ (and it is obvious for derivatives of integer-orders $n \in \mathbb{N}$ greater than one).

A correct form of the Leibniz rule for local fractional derivatives should be obtained from the fractional generalization of the Leibniz rule for the Riemann-Liouville derivatives that has the form of the infinite series. The symmetrized expression of this rule is

$$
{ }_{a}^{R L} \mathcal{D}_{x}^{\alpha}(f g)(x)=\sum_{k=0}^{\infty}\left(\begin{array}{l}
k \\
\alpha
\end{array}\right)\left(\left({ }_{a}^{R L} \mathcal{D}_{x}^{\alpha-k} f\right)(x)\left(D^{k} g(x)\right)+\left({ }_{a}^{R L} \mathcal{D}_{x}^{\alpha-k} g\right)(x)\left(D^{k} f(x)\right)\right),
$$

where the $f(x)$ and $g(x)$ are analytic functions on the interval $[a, b]$ (Theorem 15.1 of [1]), $D^{k} f(x)=f^{(k)}(x)$, and

$$
\left(\begin{array}{l}
k \\
\alpha
\end{array}\right)=\frac{\Gamma(\alpha+1)}{2 \Gamma(\alpha-k+1) \Gamma(k+1)} .
$$

It should be noted that the sum of (18) is infinite and it contains integrals of fractional order for the values $k>[\alpha]+1$. Equation (18) has been proposed by Liouville [21] in 1832. Note that extensions of the Leibniz rule to fractional derivatives are suggested by Osler [22-25].

The left-sided Riemann-Liouville fractional derivative of order $\alpha(n-1<\alpha \leq n)$ of a function $f(x)$ (see Eqs. (2.4.6) of [2]) can be presented via the Caputo fractional derivative by

$$
\left({ }_{a}^{R L} \mathcal{D}_{x}^{\alpha} f\right)(x)=\left({ }_{a}^{C} \mathcal{D}_{x}^{\alpha} f\right)(x)+\sum_{k=0}^{n-1} \frac{f^{(k)}(a)}{k !}(x-a)^{k-\alpha}, \quad(n-1<\alpha \leq n) .
$$

Substitution of Eq. (19) for $\left({ }_{a}^{R L} \mathcal{D}^{\alpha} f g\right)(x),\left({ }_{a}^{R L} \mathcal{D}^{\alpha} f\right)(x)$ and $\left({ }_{a}^{R L} \mathcal{D}^{\alpha} g\right)(x)$ into Eq. (18) gives a generalized Leibniz rule for the Caputo fractional derivatives. The extension of the Leibniz rule for the Caputo fractional derivatives also has been suggested by Theorem 3.17 of the Diethelm's book [20].

Let us give some remarks about Leibniz rule (17) for local fractional derivatives on a set of non-differentiable functions. It should be noted that statement $[26,27]$ that the Leibniz rule in the unviolated form (17) holds for non-differentiable functions is incorrect [8]. Let us give some explanations below.

(1) It should be noted that the Leibniz rule (17) cannot be used for non-differentiable functions that are not fractional-differentiable since the derivatives $\mathcal{D}^{\alpha} f(x), \mathcal{D}^{\alpha} g(x)$ and $\mathcal{D}^{\alpha}(f(x) g(x))$ should exist. 
(2) It is easy to see that nowhere in the proofs proposed in [26,27], the requirement that the functions $f(x)$ and $g(x)$ are not classically differentiable is not used. Therefore, using the same proofs, we can get the statement that the Leibniz rule (17) holds for fractionaldifferentiable functions $f(x), g(x)$ without the useless assumption that these functions are not classically differentiable.

(3) The Caputo fractional derivatives ${ }_{a}^{C} D_{x}^{\alpha}$ can be defined for functions belonging to the space $A C^{n}[a, b]$ of absolutely continuous functions [2]. If $f(x) \in A C^{n}[a, b]$, then the Caputo fractional derivatives exist almost everywhere on $[a, b]$. If $f(x) \in A C^{n}[a, b]$, then this functions is $n$-differentiable almost everywhere on $[a, b]$. The Leibniz rule (17) holds on a set of differentiable functions only for $\alpha=1$.

As a result, we get that the Leibniz rule (17) with local fractional derivative for $f(x), g(x) \in A C^{n}[a, b]$ holds only for $\alpha=1$ almost everywhere on $[a, b]$. In the countable number of points the Leibniz rule is not performed since the derivatives $\mathcal{D}^{\alpha} f(x), \mathcal{D}^{\alpha} g(x)$ and $\mathcal{D}^{\alpha}(f(x) g(x))$ does not exist. Note that the Leibniz rule for fractional derivatives of orders $\alpha=n>1(n \in \mathbb{N})$ cannot have the form (17). It should be get the rule in the form (15).

\section{Conclusion}

We prove that local fractional derivatives of differentiable functions are integer-order derivatives or zero operator. We show that definition of local fractional derivative of non-integer order $\alpha(n-1<\alpha \leq n)$ can be represented by limit of the Caputo fractional derivative of order $\alpha$. The Caputo derivative of fractional order $\alpha$ of a function $f(x)$ is defined as an action of derivative of integer order $n$ and then the integration of non-integer order $n-\alpha$. Therefore the function $f(x)$ should be classically differentiable $n$ times, and derivative $f^{(n)}(x)$ of integer order $n$ should exist. As a result, the local fractional derivative cannot be considered as the best tool for description of nowhere differentiable functions and fractal objects despite the motivation to consider these derivatives. We also argued that unviolated Leibniz rule cannot hold for local fractional derivatives of orders $\alpha \neq 1$.

A problem of strict formulation of "fractal" analogs of derivatives and integrals by extension of the classical and fractional calculus to nowhere differentiable functions and fractal sets is very difficult. We can assume that the most promising approaches to fractional calculus on fractals can be based on methods of "non-integer dimensional spaces" (see $[28,29]$ and references therein) or "analysis on fractals" [30-33].

\section{References}

1. Samko, S.G., Kilbas, A.A., Marichev, O.I.: Integrals and Derivatives of Fractional Order and Applications (Nauka i Tehnika, Minsk, 1987); and Fractional Integrals and Derivatives Theory and Applications. Gordon and Breach, New York (1993)

2. Kilbas, A.A., Srivastava, H.M., Trujillo, J.J.: Theory and Applications of Fractional Differential Equations. Elsevier, Amsterdam (2006)

3. Podlubny, I.: Fractional Differential Equations. Academic Press, San Diego (1998)

4. Ross, B.: A brief history and exposition of the fundamental theory of fractional calculus, in fractional calculus and its applications. In: Proceedings of the International Conference Held at the University of New Haven, June 1974 Series: Lecture Notes in Mathematics. vol. 457, pp. 1-36. Springer, Heidelberg (1975)

5. Tenreiro Machado, J., Kiryakova, V., Mainardi, F.: Recent history of fractional calculus. Commun. Nonlinear Sci. Numer. Simul. 16(3), 1140-1153 (2011) 
6. Tenreiro Machado, J.A., Galhano, A.M.S.F., Trujillo, J.J.: On development of fractional calculus during the last fifty years. Scientometrics $\mathbf{9 8}(1), 577-582(2014)$

7. Tarasov, V.E.: No violation of the Leibniz rule. No fractional derivative. Commun. Nonlinear Sci. Numer. Simul. 18(11), 2945-2948 (2013). arXiv:1402.7161

8. Tarasov, V.E.: Comments on The Minkowski's space-time is consistent with differential geometry of fractional order, [Physics Letters A 363 (2007) 5-11]. Phys. Lett. A 379(14-15), 1071-1072 (2015)

9. Mainardi, F.: Fractional Calculus and Waves in Linear Viscoelasticity: An Introduction to Mathematical Models. World Scientific, Singapore (2010)

10. Tarasov, V.E.: Fractional Dynamics: Applications of Fractional Calculus to Dynamics of Particles, Fields and Media. Springer, New York (2011)

11. Uchaikin, V.V.: Fractional Derivatives for Physicists and Engineers. Volume II. Applications. Springer, New York (2012)

12. Kolwankar, K.M., Gangal, A.D.: Fractional differentiability of nowhere differentiable functions and dimension. Chaos 6(4), 505-513 (1996). arXiv:chao-dyn/9609016

13. Kolwankar, K.M., Gangal, A.D.: Hölder exponents of irregular signals and local fractional derivatives Pramana. J. Phys. 48(1), 49-68 (1997). arXiv:chao-dyn/9711010

14. Kolwankar, K.M., Gangal, A.D.: Local fractional Fokker-Planck equation. Phys. Rev. Lett. 80(2), 214-217 (1998). arXiv:cond-mat/9801138

15. Kolwankar, K.M.: Local fractional calculus: a review. arXiv:1307.0739

16. Caputo, M.: Linear models of dissipation whose $\mathrm{Q}$ is almost frequency independent-II. Geophys. J. R. Astron. Soc. 13(5), 529-539 (1967). [Reprinted. In: Fractional Calculus and Applied Analysis. Vol. 11. No.1. (2008) 3-14.]

17. Caputo, M.: Elasticita e Dissipazione. Zanichelli, Bologna (1969). (in Italian)

18. Caputo, M., Mainardi, F.: Linear models of dissipation in anelastic solids. Riv. del Nuovo Cim. 1(2), 161-198 (1971). (Serie II)

19. Caputo, M., Mainardi, F.: A new dissipation model based on memory mechanism. Pure Appl. Geophys. 91(1), 134-147 (1971)

20. Diethelm, K.: The Analysis of Fractional Differential Equations: An Application-Oriented Exposition Using Differential Operators of Caputo Type. Springer, Heidelberg (2010)

21. Liouville, J.: Memoire sur le calcul des differentielles a indices quelconques. J. l'Ec. R. Polytech. 13, 71-162 (1832)

22. Osler, T.J.: Leibniz rule for fractional derivatives generalized and an application to infinite series. SIAM J. Appl. Math. 18(3), 658-674 (1970)

23. Osler, T.J.: Fractional derivatives and Leibniz rule. Am Math. Mon. 78(6), 645-649 (1971)

24. Osler, T.J.: A further extension of the Leibniz rule to fractional derivatives and its relation to Parseval's formula. SIAM J. Math. Anal. 3(1), 1-16 (1972)

25. Osler, T.J.: A correction to Leibniz rule for fractional derivatives. SIAM J. Math. Anal. 4(3), 456-459 (1973)

26. Ben, F., Cresson, A.J.: About non-differentiable functions. J. Math. Anal. Appl. 263, 721-737 (2001)

27. Babakhani, A.: On calculus of local fractional derivatives. J. Math. Anal. Appl. 270(1), 66-79 (2002)

28. Tarasov, V.E.: Vector calculus in non-integer dimensional space and its applications to fractal media. Commun. Nonlinear Sci. Numer. Simul. 20(2), 360-374 (2015). arXiv:1503.0202

29. Tarasov, V.E.: Anisotropic fractal media by vector calculus in non-integer dimensional space. J. Math. Phys. 55(8), 083510 (2014). arXiv:1503.0239

30. Kugami, J.: Analysis on Fractals. Cambridge University Press, Cambridge (2001)

31. Strichartz, R.S.: Differential Equations on Fractals. Princeton University Press, Oxford (2006)

32. Harrison, J.: Flux across nonsmooth boundaries and fractal Gauss/Green/Stokes' theorems. J. Phys. A 32(28), 5317-5328 (1999)

33. Kumagai, T.: Recent developments of analysis on fractals. In: Roussas, G. (ed.) Selected Papers on Analysis and Related Topics. American Mathematical Society Translations, vol. 2, pp. 81-96. Kluwer Academic Publishers, New York (2008) 